\title{
NIVEL DE SOBRECARGA EN EL DESEMPEÑO DEL ROL DEL CUIDADOR FAMILIAR DE ADULTO MAYOR CON DEPENDENCIA SEVERA ${ }^{1}$
}

\author{
BURDEN LEVEL IN PERFORMING THE ROLE OF FAMILY \\ CAREGIVERS OF ELDERLY WITH SEVERE DEPENDENCE
}

\author{
ElizABeth Flores G. \\ EDITH Rivas R. ${ }^{*}$ \\ Fredy Seguel P.**
}

\begin{abstract}
RESUMEN
Objetivo: Determinar el nivel de sobrecarga en el desempeño del rol, en el cuidador familiar de adulto mayor con dependencia severa y su relación con características sociodemográficas. Material y métodos: Estudio de corte transversal, correlacional y predictivo, en 67 cuidadores familiares adscritos a Programas de Postrados. Se utilizó Escala de Zarit y Encuesta Sociodemográfica. Resultados: Los cuidadores son mujeres, hijas, casadas, media de edad 58,6 años, condición socioeconómica baja, sin ocupación, escolaridad media incompleta, llevan 1 - 5 años cuidando, dedican 21 - 24 h diarias, sin actividad recreativa, sin ayuda de otras personas y reconocen el apoyo de Centros de Salud Comunitarios. Más de la mitad presentan sobrecarga intensa. La variable sobrecarga del cuidador sólo se asoció significativamente a instituciones que apoyan al cuidador y es factor predictivo de la sobrecarga. Conclusión: El cuidado de las personas mayores se puede sobrellevar con la organización del trabajo familiar en forma igualitaria y equitativa. Ratifica a la enfermería comunitaria y las organizaciones sociales como factor amortiguador de sobrecarga del cuidador familiar. Se requiere de iniciativas y proyectos de desarrollo social que aumenten las redes de apoyo, además, mejorar el plan de apoyo al cuidador con dependencia severa que existe en Chile.
\end{abstract}

Palabras clave: Envejecimiento, anciano frágil, cuidadores de familia.

\begin{abstract}
Objective: To determine the level of burden on role performance in family caregivers of older adults with severe dependence and its relationship to sociodemographic characteristics. Methodology: A cross-sectional, correlational and predictive, of 67 family as caregivers assigned to postrate programs. Zarit Scale was used and Sociodemographic Survey. Results: Family as caregivers are women, daughters, married, mean age 58.6 years, lower socioeconomic status, without occupation, incomplete secondary schooling, are 1 to 5 years caring, dedicated 21 to $24 \mathrm{~h}$ a day, no recreational activity without help of others and recognize the support of Community Health Centers. More than half have burden. The only variable caregiver burden was significantly associated with institutions that support the caregiver and to predict the overload. Conclusion: The care of older people can only cope with the organization of family labor equally and fairly. Ratifies community nursing and social

\footnotetext{
${ }^{1}$ Publicación derivada de Tesis de Magíster en Enfermería, mención Gestión del Cuidado, Universidad de La Frontera, Temuco, Chile.

*Enfermera, Docente Universidad Austral de Chile, Valdivia, Chile. Email: idaflores@uach.cl

${ }^{* *}$ Enfermera, Docente Universidad de la Frontera, Temuco, Chile. Email: erivas@ufro.cl

${ }^{* * *}$ Enfermero, Docente Universidad Austral de Chile, Valdivia, Chile. Email: fredyseguel@uach.cl
} 
organizations as shock factor family caregiver overload. It requires initiatives and projects that enhance social support networks, as well as improve the caregiver support plan with severe dependence that exists in Chile.

Key words: Aging, frail elderly, caregivers.

Fecha recepción: 25/04/11 Fecha aceptación: 26/03/12

\section{INTRODUCCIÓN}

A nivel mundial existe una tendencia global de envejecimiento de la población, de la que Chile no está ajeno. Si bien es un elemento natural de la vida, el proceso de transición demográfica ha ocurrido de forma acelerada. El grupo de edad de mayores de 60 años tiene cada vez más peso relativo, alcanzando el 11,4\% del total de la población, de los cuales aproximadamente el 15\% tiene más de 80 años (1). Situación que, sumada al escaso mejoramiento de los estilos de vida saludable, trae consigo el aumento de los adultos mayores postrados (AMP) o con dependencia severa, que en Chile se estima entre un 4 y $7 \%$ (1). Esto conlleva a una pérdida de autonomía en los Adultos Mayores (AM), surge entonces la necesidad de cuidado, de contar con ayuda de otras personas para satisfacer las demandas (2), potencial que se desarrolla según la situación a las que se vean enfrentadas las personas (3). Los sistemas sanitarios tratan de satisfacer las necesidades de cuidado de salud enfocando su atención en términos de mantenimiento de la capacidad funcional (4). Cuidar será siempre indispensable para la perpetuidad de la sociedad, es el pilar de la profesión de enfermería, pero también tiene un componente no profesional, el cuidado familiar en el domicilio, que es considerado una obligación moral (5).

\section{Cuidador familiar}

Los cuidadores familiares son personas que otorgan cuidado informal, no reciben remuneración o retribución económica, se catalo- gan como principales o primarios y secundarios, según el grado de responsabilidad en el cuidado de los ancianos (6) y son definidos como "aquella persona que asiste o cuida a otra afectada de cualquier tipo de discapacidad, minusvalía o incapacidad que le dificulta o impide el desarrollo normal de sus actividades vitales o de sus relaciones" (7).

La experiencia de cuidar involucra una gran responsabilidad y un enorme esfuerzo, provoca un aumento en la carga del cuidador, la cual si no se logra manejar adecuadamente puede traer repercusiones físicas, mentales y socioeconómicas y, por lo tanto, comprometer la vida, la salud, el bienestar, el cuidado y autocuidado no sólo del cuidador, sino también de la persona dependiente de cuidados, por lo que se requiere de un soporte social más global (4). Cuidar a una persona con discapacidad o enfermedad crónica "implica ver la vida de una manera diferente, modificar las funciones a las que se está acostumbrado, tomar decisiones en medio de alternativas complicadas, asumir responsabilidades o realizar tareas y acciones de cuidado físico, social, psicológico y religioso para atender las necesidades cambiantes de la persona cuidada" (8).

Cuando se requiere de cuidados con mayor complejidad, tiempo y dedicación, se está frente a cuidados extraordinarios, que implican una mayor responsabilidad, gasto de energía y tiempo, es decir representan cuidados que sobrepasan los límites de lo considerado usual. Este cuidado a veces puede durar meses o años, puede exigir gran esfuerzo físico, suelen ser desagradables y provocan interrupción de los roles que desempeña la persona tanto en su familia como en la sociedad. 
Esta responsabilidad la asumen en forma voluntaria o porque no existe otra alternativa $y$, además, sin una recompensa económica. El cuidador principal se caracteriza por ser mujer, con lazos de parentesco, generalmente la esposa o una hija, que asume el rol de cuidador de manera informal y voluntario (9). Constituye una unidad social sujeta a las presiones y condiciones de su entorno cultural, económico y político de un momento dado y se encarga de brindarle apoyo social, funcional, económico o material, afectivo y asistencia en diversas formas (10). Po lo anterior, los cuidadores pueden experimentar una variada gama de emociones, como ira, culpa, frustración, agotamiento, ansiedad, miedo, dolor, tristeza, amor, aumento de la autoestima y satisfacción del trabajo realizado, porque dependiendo de las circunstancias individuales en que se asume el rol de cuidador, puede significar un cambio brusco para el que no se está preparado y originar sentimientos de pérdida importante, de lo que fue antes de asumir el rol de cuidador y de las expectativas que se había trazado (11).

Los recursos familiares para entregar los cuidados se ven disminuidos; la persona que otorga los cuidados también es de edad avanzada, porque mayoritariamente se trata del cónyuge y puede tener discapacidades que limitan el tipo de ayuda que puede proporcionar. Las familias son pequeñas por lo que hay menos personas con quién compartir el cuidado, la participación de las mujeres en el trabajo hace que dispongan de menos tiempo para otorgar asistencia, la mayor cantidad de divorcios también ha debilitado los lazos familiares y puede disminuir la ayuda disponible cuando un padre se vuelve discapacitado $y$, también, los hijos de padres divorciados pueden tener menos recursos para comprometerse con el cuidado de sus padres ancianos (12).

\section{Dependencia}

El cuidado familiar ha emergido como un tema importante en el ámbito clínico y social, porque "la dependencia de un cónyuge o padre puede también intensificar problemas de larga data al interior de la familia, o abrumar los recursos fisiológicos del cuidador" (12).

El Consejo de Europa (13) define la dependencia como "un estado en el que se encuentran las personas que por razones ligadas a la falta o pérdida de autonomía física, psíquica o intelectual tienen necesidad de asistencia y/o ayudas importantes a fin de realizar los actos corrientes de la vida diaria". En Chile el Servicio Nacional del Adulto Mayor, SENAMA (14), estipula que las definiciones de dependencia se enmarcan "dentro de las limitaciones o incapacidad para efectuar las actividades de la vida diaria", y Barthel la describe como "la incapacidad funcional en relación con las actividades de la vida diaria"(14). Se considera una situación específica de discapacidad, observándose una limitación severa de la persona para realizar una determinada actividad y la necesidad de recibir ayuda personal o técnica para poder interactuar con el medio ambiente, por lo que la relación discapacidad y dependencia constituye una situación compleja de salud (14).

En Chile, las políticas ministeriales incluyen la prevención de la pérdida de funcionalidad, la rehabilitación comunitaria y los cuidados domiciliarios del AMP. Esta última es considerada una tarea difícil, debido a la pobreza y déficit de conocimientos del cuidador, y a la falta de recursos a nivel de los centros de salud comunitarios para realizar el seguimiento, por lo que las redes de apoyos sociales y familiares adquieren especial importancia (15). El programa contiene la Medida 6B, que establece capacitación, apoyo y asistencia técnica de un equipo de salud, a través de visitas domiciliarias, que vela por la condición y calidad del cuidado al interior del hogar en que reside la persona. Tiene como fin mejorar la calidad del cuidado de la persona postrada y aliviar a la familia cuidadora, disminuyendo la carga del cuidador e impidiendo de este modo la institucionalización (16). 


\section{Carga del cuidador}

Definida como la experiencia subjetiva del cuidador de las demandas de atención, es un concepto multidimensional, con características objetivas y subjetivas. La carga objetiva corresponde a los cambios que debe realizar el cuidador en diversos ámbitos de la vida y la carga subjetiva se refiere a las reacciones emocionales frente a las demandas de cuidado (17). Para dimensionar los efectos emocionales adversos que manifiesta el cuidador frente a la rutina diaria de cuidar a una persona, es decir la carga del cuidador, existen diversas pruebas, una de las más utilizadas es la Zarit Burden Interview, que considera factores emocionales, físicos, las finanzas, la actitud del cuidador hacia el receptor de los cuidados, la relación entre ellos, los comportamientos y actitudes expresadas por el receptor de cuidados, por lo que esta escala mide el riesgo de deterioro de su vida social, laboral, familiar, los problemas económicos y el sentimiento de sobrecarga en el rol de cuidar (18). El cuidador de un familiar con dependencia severa debe enfrentar momentos críticos relacionados con el estrés de cuidar, el agotamiento, la falta de apoyo de otros miembros de la familia que en muchas ocasiones actúan como jueces, la reducción del número de vínculos, disminución de las oportunidades, la motivación y la energía para establecer y mantener relaciones sociales lo que los predispone a presentar el Síndrome del Cuidador (10), conocido en el ámbito de la enfermería como Cansancio en el Rol del Cuidador, por lo que es importante contar con redes de apoyo familiares, amistades o recursos de la sociedad (19).

Frecuentemente el cuidador ve afectado su estado de salud, su bienestar y se siente incapaz de afrontar esta responsabilidad, pasando a constituir para el área sanitaria un paciente "oculto" o "desconocido", que requiere de un diagnóstico precoz e intervención inmediata, antes de presentar el Síndrome del Cuidador (20). Asimismo, se sienten agobia- dos por las exigencias de cuidados, llegando a presentar ansiedad y depresión. Estos efectos son mayores y más frecuentes en cuidadores que deben lidiar con personas que presentan problemas conductuales, como agresividad y conducta errante, además cuando el curso de la enfermedad es de naturaleza impredecible. Pero también se han descrito efectos positivos asociados al cuidado, como el aumento de la autoestima, mayor satisfacción personal al sentir que el ser querido está siendo cuidado adecuadamente (21). No obstante lo anterior, hay autores que han observado que cuidar por tiempo prolongado permite a los cuidadores ir realizando una adecuación en sus rutinas, de manera de lograr una adaptación paulatina al rol, lo que va aminorando los efectos negativos y disminuye la probabilidad de sufrir estrés crónico (17).

Zarit (12) menciona que son las familias las que con mayor frecuencia se encargan del cuidado de los ancianos dependientes, durante periodos prolongados, para hacer frente a una sociedad que envejece. El autor considera que la responsabilidad no puede recaer sólo en la familia; por la intensidad y costo de las cargas sugiere una responsabilidad compartida, creativa, que se consideren las necesidades de la persona cuidada y del cuidador, por lo que es necesario realizar estudios que conduzcan al logro de mejores estrategias de intervención.

Un estudio cualitativo de calidad de vida de los cuidadores familiares de adultos mayores con dependencia funcional, menciona la soledad, el aislamiento social, la sobrecarga de trabajo, el deterioro de la salud física y mental, y el olvido de sí mismo, como consecuencia del desempeño del rol de cuidador (22). Existen escasos y poco concluyentes estudios que determinan si la condición de dependencia de la persona cuidada se relaciona en forma directa con la sobrecarga percibida por el cuidador; al respecto Ocampo et al. mencionan que "Zarit et al. no encontraron asociación entre el deterioro en las Actividades Básicas Cotidianas (ABC) físicas y la car- 
ga del cuidador" (23).

Debido a que los cuidadores representan un recurso muy valioso para alcanzar el bienestar de la población que cuidan y que el desempeño de este rol les puede provocar consecuencias negativas para su salud, surge la necesidad de conocer desde la perspectiva de quienes cuidan, cuál es el nivel de sobrecarga en el desempeño del rol y cuáles son los factores relacionados para poder establecer estrategias de intervención que permitan prevenir o detectar precozmente la sobrecarga en los cuidadores familiares.

El objetivo de este estudio es determinar la relación entre la carga percibida y el perfil sociodemográfico de los cuidadores familiares de adultos mayores con dependencia severa.

\section{MATERIAL Y MÉTODO}

Se planteó una investigación de corte transversal, correlacional y predictiva (24). La población de estudio fueron 67 cuidadores familiares de adultos mayores con dependencia severa, adscritos al Programa de Postrados del Consultorio Externo y al CESFAM Dr. Jorge Sabat de Valdivia, que corresponde a la totalidad de los cuidadores que cumplieron con los criterios de inclusión. Se incluyeron cuidadores familiares que tenían más de 3 meses en el rol de cuidador y que aceptaron participar, explicitado en el Consentimiento Informado. La recolección de datos se realizó a través de la Escala de Carga del Cuidador de Zarit, y se adicionó una Encuesta de Perfil Sociodemográfico. Esta escala intenta objetivar la carga del cuidador, mide el riesgo de deterioro de la vida social, laboral, familiar, los problemas económicos y el sentimiento de sobrecarga en el rol de cuidar; en Chile, el instrumento fue validado en el año 2008 por Breinbauer et al. (25) en sus versiones original y abreviada, en una muestra de 32 cuidadores de pacientes postrados del Centro de
Salud San Manuel de Melipilla, Chile.

La recolección de los datos fue realizada por el equipo investigador a través de visita domiciliaria al 100\% de los cuidadores de adultos mayores con dependencia severa (67 cuidadores), previo consentimiento informado. La duración de la visita fue de $1 \mathrm{~h}$ y el periodo de recolección comprendió los meses de agosto y septiembre del año 2010.

En el análisis de los datos se utilizaron estadísticos descriptivos, paramétricos y no paramétricos como prueba exacta de Fischer para variables categóricas, de correlación para analizar las relaciones entre las variables y predictivas, como la regresión lineal simple, tomando como variable dependiente la sobrecarga del cuidador y como variables independientes o predictivas las sociodemográficas, sin embargo, sólo se realizó un modelo tomando como variable independiente el número de instituciones que apoyan al cuidador, porque fue la única variable que presentó correlación. Se comprobaron los supuestos de la regresión lineal de normalidad, especificidad, datos influyentes y errores. El análisis se apoyó en el programa SPSS de Windows, versión 11.5.

Las variables utilizadas fueron: sexo, vinculación con el adulto mayor, estado civil, previsión, situación laboral, persona que le ayuda en el rol de cuidador, instituciones u organizaciones que lo apoyan, presencia o ausencia de enfermedades, religión, etnia, actividad recreativa, tiempo en el rol de cuidador, horas diarias dedicadas al cuidado, ingresos económicos familiares y nivel de sobrecarga del cuidador y edad, número de años de estudio, número de personas que viven en el hogar, número de personas que le ayudan en el rol de cuidador, sobrecarga del cuidador.

Implicancias éticas: el estudio se sometió al comité de ética del Servicio de Salud Valdivia, se consideraron los principios de la bioética para equilibrar los beneficios frente a los riesgos, evitar hacer daño y respetar la capacidad de decisión de las personas (26). Con el objeto de asegurar que los derechos 
de las personas que participaron en el estudio fueran protegidos, se efectuó consentimiento informado. Respecto de los criterios de rigor ético se consideraron: Valor de Verdad, Aplicabilidad, Consistencia y Neutralidad, para dar fe de rigurosidad y veracidad de los resultados del estudio. Conjuntamente, se plantea la claridad del investigador en relación a las implicancias del estudio para ser expuestas detalladamente al participante, en forma previa, con el fin de escoger libremente sobre su participación en él (27).

\section{RESULTADOS}

La población correspondió en un 55,2\% al Centro de Salud Familiar Dr. Sabat y en un $44,8 \%$ al Consultorio Externo de Valdivia. Se observó predominio del sexo femenino $(91 \%)$, mayoritariamente correspondía a hijas/os (49,3\%), con media de edad de 58,6 años, 85,2\% eran mayores de 45 años, que no realizaban trabajo remunerado fuera del hogar. La mayoría son casados $(50,7 \%)$, de bajos ingresos económicos, su situación previsional indica que son mayoritariamente carentes de recursos e indigentes o con ingreso imponible mensual menor o igual a $\$ 172.000$. El 64,2\% profesaban religión católica, 22\% refirieron no contar con personas que le ayuden en el rol de cuidar, el $29 \%$ sólo cuenta con 1 ó 2 personas que les colaboran, además el 46,3\% de los cuidadores refirió dedicar 21 a $24 \mathrm{~h}$ del día al cuidado. Cabe destacar que el 43,3\% manifiesta llevar de 1 a 5 años desempeñando el rol de cuidador, el 92,5\% refiere recibir apoyo de las instituciones de salud y muchos de los cuidadores presentaban alguna patología, 3 de cada 4 cuidadores refieren no realizar actividades recreativas y la etnia mapuche sólo alcanzó el 13,4\% (Tabla 1).

Tabla 1. Caracterización del cuidador familiar según variables sociodemográficas.

\begin{tabular}{lcc}
\hline Variable & Ítemes & $\%$ \\
\hline \multirow{2}{*}{ Sexo } & Femenino & 91 \\
& Masculino & 9 \\
\hline \multirow{4}{*}{ Estado civil actual } & Soltero & 31,3 \\
& Casado & 50,7 \\
& Conviviente & 1,5 \\
& Separado & 6 \\
& Divorciado & 7,5 \\
& Viudo & 3 \\
\cline { 2 - 3 } Edad (años) & $15-25$ & 1,5 \\
\cline { 2 - 3 } & $26-35$ & 0 \\
\cline { 2 - 3 } & $36-45$ & 13,4 \\
\cline { 2 - 3 } & $46-55$ & 29,9 \\
\cline { 2 - 3 } & $56-65$ & 25,4 \\
\cline { 2 - 3 } & $>65$ & 29,9 \\
\hline
\end{tabular}




\begin{tabular}{lcc} 
Continuación Tabla 1. & & \\
\hline & 1 & 22 \\
Número de personas & 2 & 19 \\
que ayudan & 3 & 10 \\
& 4 & 11 \\
& 5 a 9 & 3 \\
Tiempo en rol & 3meses a 1 año & 2 \\
\hline & 1 a 5 años & 43,5 \\
& 5 a 10 años & 22,4 \\
Horas diarias de & 10 a 15 años & 14,9 \\
cuidado & Más de 15 años & 11,9 \\
& 0 a 5 & 6 \\
& 6 a 10 & 13,4 \\
Instituciones que & 11 a 15 & 9 \\
apoyan & 16 a 20 & 25,4 \\
& 21 a 24 & 46,3 \\
\hline & Salud & 92,5 \\
Vinculación & Comunitarias & 13,4 \\
& Estatales & 19,4 \\
\hline & Cónyuge & 23,9 \\
& Hijo/a & 49,3 \\
& Hermano/a & 6 \\
& Nuera/ Yerno & 6 \\
& Sobrino/a & 6 \\
& Otro & 9 \\
\hline & & \\
& &
\end{tabular}

Respecto de la sobrecarga, 6 de cada 10 carga ligera (23,9\%) y 16,4\% no presentaba cuidadores presentaba sobrecarga intensa sobrecarga, la media fue de 62,8 pts., es decir $(59,7 \%)$, la cuarta parte presentaba sobre- sobrecarga intensa (Tabla 2).

Tabla 2. Caracterización de los cuidadores familiares de adultos mayores con dependencia severa, según niveles de sobrecarga.

\begin{tabular}{cccccccc}
\hline Caracterización & Ítemes $^{\star}$ & $\%$ & Media & Mediana & DS & Mínimo & Máximo \\
\hline \multirow{3}{*}{ Sobrecarga en rol } & Ausencia & 16,4 & & & & & \\
& Ligera & 23,9 & 62,6 & 63 & 15,9 & 35 & 98 \\
& Intensa & 59,7 & & & & & \\
\hline
\end{tabular}

*Ausencia: $<46$ puntos, Ligera: $47-55$ puntos, Intensa $\geq 56$ puntos. 
Al estudiar la asociación de la sobrecarga del cuidador con las variables sociodemográficas, no resultó significativa con: sexo, etnia, religión que profesa, previsión, situación laboral, nivel de ingresos económicos familiares, realización de actividades recreativas, tiempo en la labor de cuidar. No obstante, en términos porcentuales se observó menor sobrecarga en los grupos extremos, es decir, los que llevaban de 3 meses a 1 año $(60 \%)$ y el grupo de más de 15 años dedicados al cuidado (50\%) (Tabla 3).

Tabla 3. Relación entre el nivel de sobrecarga y tiempo de cuidador.

\begin{tabular}{ccccccccc}
\hline \multirow{2}{*}{$\begin{array}{c}\text { Tiempo de } \\
\text { cuidador }\end{array}$} & \multicolumn{2}{c}{$\begin{array}{c}\text { Ausencia } \\
\text { sobrecarga }\end{array}$} & \multicolumn{2}{c}{ Sobrecarga ligera } & Sobrecarga intensa & \multicolumn{2}{c}{ Total } \\
\cline { 2 - 8 } & $\mathbf{N}^{\circ}$ & $\%$ & $\mathbf{N}^{\circ}$ & $\%$ & $\mathbf{N}^{\circ}$ & $\%$ & $\mathbf{N}^{\circ}$ & $\%$ \\
\hline $\begin{array}{c}3 \text { meses }-1 \\
\text { año }\end{array}$ & 0 & 0 & 3 & 60 & 2 & 40 & 5 & 100 \\
$1-5$ años & 6 & 20,7 & 4 & 13,8 & 19 & 65,5 & 29 & 100 \\
$6-10$ años & 4 & 26,7 & 1 & 6,7 & 10 & 66,7 & 15 & 100 \\
$11-15$ años & 0 & 0 & 5 & 50 & 5 & 50 & 10 & 100 \\
Más de 15 & 1 & 12,5 & 3 & 37,5 & 4 & 50 & 8 & 100 \\
años & 11 & 16,4 & 16 & 23,9 & 40 & 59,7 & 67 & 100 \\
\hline Total & & & & & & &
\end{tabular}

p: no significativa.

Las pruebas estadísticas indican que no es significativa la asociación entre sobrecarga del cuidador y número de horas diarias dedicadas al cuidado de adultos mayores con dependencia severa $(\mathrm{p}=0,17)$, a pesar de ello, se observó que sólo el grupo de 1 a 5 h diarias dedicadas al cuidado no presentó sobrecarga intensa, en todos los demás grupos se observaron similares niveles de sobrecarga.

Al estudiar la correlación de la variable sobrecarga global en el rol de cuidar y las variables numéricas número de personas que ayudan, número de personas que viven en el hogar, años de estudio y edad del cuidador, los resultados mostraron que la sobrecarga global del cuidador no presenta correlación con ninguna variable.

$\mathrm{Al}$ utilizar las variables instituciones que apoyan y sobrecarga global, se creó un modelo que resultó significativo $(\mathrm{p}=0,042)$, donde el número de instituciones que apoyan al cuidador es un factor predictivo de la sobrecarga, encontrándose que aquellos cuidadores que reciben ayuda de 2 o más instituciones, tienen 8,6 puntos menos de sobrecarga que los que tienen una y ninguna institución que los apoyen (Tabla 4).

\section{DISCUSIÓN}

En el estudio se encontró que el 59,7\% de los cuidadores presentaba sobrecarga intensa, lo que difiere mucho de los estudios revisados, donde los autores han descrito que sólo el 5,8\% de los cuidadores presentaba sobrecarga intensa $(23,28)$. Los cuidadores continúan siendo mayoritariamente mujeres $(2$, 23,28 ), fenómeno que se puede explicar por los patrones culturales imperantes en nuestra sociedad de machismo y patriarcado, que asigna a la mujer el papel de cuidadora y principalmente el cuidado de personas de- 
Tabla 4. Influencia del número de instituciones sobre la sobrecarga global del cuidador.

\section{Modelo de regresión lineal}

Resumen del modelo

\begin{tabular}{ccccc}
\hline Modelo & $\mathbf{R}$ & R cuadrado & R cuadrado corregida & $\begin{array}{c}\text { Error típ. de la } \\
\text { estimación }\end{array}$ \\
\hline 1 & $0,25 \mathrm{a}$ & 0,06 & 0,05 & 15,54 \\
\hline
\end{tabular}

a. Variables predictoras: (Constante), una y varias Inst.

ANOVA(b)

\begin{tabular}{clccccc}
\hline \multirow{2}{*}{ Modelo } & & $\begin{array}{c}\text { Suma de } \\
\text { cuadrados }\end{array}$ & gl & Media cuadrática & F & Sig. \\
\hline \multirow{2}{*}{1} & Regresión & 1036,108 & 1 & 1036,108 & 4,291 & $0,042 \mathrm{a}$ \\
& Residual & 15694,668 & 65 & 241,456 & & \\
\hline
\end{tabular}

a.Variables predictoras: (Constante), una y varias Inst.

b.Variable dependiente: Sobrecarga global.

\begin{tabular}{cccccc}
\hline \multirow{2}{*}{ Modelo 1 } & \multicolumn{1}{c}{ Coeficientes no estandarizados } & $\begin{array}{c}\text { Coeficientes } \\
\text { estandarizados }\end{array}$ & $\mathbf{t}$ & Sig. \\
\cline { 2 - 5 } & $\mathbf{B}$ & Error típ. & Beta & B & Error típ. \\
\hline (Constante) & 68,7 & 3,475 & & 19,772 & 0 \\
\hline $\begin{array}{l}\text { Una y varias } \\
\text { Inst. Apoyan }\end{array}$ & $-8,594$ & 4,149 & $-0,249$ & $-2,071$ & 0,042 \\
\hline
\end{tabular}

pendientes, como niños, ancianos y personas con discapacidades físicas y psíquicas; además estas mujeres desempeñaban otros roles como madre de familia, esposa e hija. Respecto al vínculo con la persona cuidada, afín a lo referido por Pérez (2) y Ocampo et al. (23), la mayoría fueron hijas/os y cónyuges, lo que se relaciona con la edad de la persona cuidada (65 años y más), por lo que muchos de ellos tienen hijos y no parejas que le sobrevivan. Se trató mayoritariamente de cuidadores que no realizaban trabajo remunerado fuera del hogar, ya que debido a las demandas de cuidado de los adultos mayores, dedican gran cantidad de horas diarias a satisfacerlas y por lo tanto no disponen de tiempo para trabajar, provocándose una incompatibilidad entre la realización y cumplimiento del trabajo fuera del hogar y el cuidado del familiar, coincidiendo con investigaciones que revelan que la mayoría de los cuidadores corresponde a amas de casa sin trabajo remunerado $(2,28)$. Esta razón también se esgrime para justificar la escasa proporción de cuidadores que realizan actividad recreativa. Asimismo, se encontró que existen algunas diferencias respecto a la edad; si bien es cierto los rangos de edad coinciden con los encontrados en estudios internacionales de 24 a 87 años (23), la media fue superior, como también fue mayor a la encontrada en España (2). De igual forma, la mayoría de los cuidadores tenían más de 45 años. Según Pérez (2), esto ocurre cuando la persona receptora de los cuidados es mayor de 50 años, también se explica porque le sobreviven princi- 
palmente hijos. Referente al estado civil, afín con lo encontrado por Pérez (2) en España, este estudio reflejó que mayoritariamente se trató de cuidadores casados, seguido de los solteros, lo que va de la mano con el comportamiento general de la población chilena registrado en el Censo 2002 del Instituto Nacional de Estadísticas (INE), que indica que la condición de casados alcanza el 46,16\% de los habitantes mayores de 15 años (29). La escolaridad de los cuidadores fue superior a la observada en otras investigaciones, la media de años de estudio correspondió a 9,33; el 19,4\% tenía estudios superiores y los que no sabían leer ni escribir representaron sólo el 6\% del total de cuidadores. En este sentido, en Chile el promedio de años de escolaridad ha aumentado, alcanzando más de 10 años en mayores de 18 años, además las personas con estudios de enseñanza básica o media reciben bajas remuneraciones (270 a $350 \mathrm{mil}$ pesos mensuales), que no son suficiente estímulo para compensar el hecho de entregar el cuidado de un ser querido a otras personas y tampoco es suficiente para costear esta ayuda, por lo que la mayoría opta por dedicarse ellos mismos al cuidado de su familiar (30).

Las familias se caracterizan por ser de nivel socioeconómico bajo (31), reciben básicamente pensiones y el estipendio ${ }^{2}$ que entrega el Estado a los que cuidan a personas que tienen la calidad de postrados y que pertenecen a los estratos socioeconómicos más bajos, lo que les confiere un grado de vulnerabilidad social; se debe considerar además que gran parte de estos ingresos los destinan al cuidado, como la adquisición de insumos, alimentos y medicamentos (32).

La mayoría lleva de 1 a 5 años dedicados al cuidado, no es posible determinar la media, porque la variable fue considerada como categórica, sin embargo estudios como el realizado en Taiwan (28) muestra una media de 54 meses. En el estudio se encontró que la mayor proporción de cuidadores lleva

${ }^{2} 20.000$ pesos chilenos, equivalentes a US\$ 41 . de 1 a 5 años dedicados al cuidado y dedican entre 21 y $24 \mathrm{~h}$ al día. Al respecto Ocampo et al. (23) también refieren que la mayor frecuencia corresponde al rango de 20 a $24 \mathrm{~h}$ al día, pero con una proporción de cuidadores muy superior $(74,2 \%)$. Chiou et al. (28) mencionan que los cuidadores dedican 14,3 h diarias al cuidado, debido a la condición de dependencia severa presente en todos los adultos mayores, quienes tienen una declinación de sus capacidades funcionales y de su autonomía, que no les permite realizar las actividades de la vida diaria, tan básicas y necesarias como alimentarse, bañarse, asearse e ir al baño (14), siempre requieren de la ayuda de otra persona, dedicando mucho tiempo en la realización de estas actividades y además estar constantemente presente para satisfacer cualquier necesidad que surja en su ser querido. En el estudio se observó que la mayoría de los cuidadores no contaban con personas que les ayuden en el cuidado y que sólo recibía ayuda de un familiar. Las personas que más colaboraban en las labores del cuidado son miembros de la misma familia, esto es consecuente con lo mencionado por Zarit (12), respecto a que históricamente la familia ha sido la principal fuente de ayuda para los ancianos y personas con algún grado de dependencia; esta situación puede incidir en el cumplimiento de las responsabilidades familiares y laborales. Los encuestados reconocieron a los centros de salud como entidad prestadora de servicios, aunque refieren que la frecuencia de las visitas aún dista de ser la necesaria, en este sentido Zarit et al. (33) concluyeron en su estudio que el apoyo de los servicios sanitarios no fue significativo en la sobrecarga del cuidador.

Respecto a que los cuidadores frecuentemente ven afectado su estado de salud y su bienestar debido a la situación de estrés, de no sentirse capaz de afrontar la situación y porque cuidar implica realizar tareas complejas que exigen un gran esfuerzo físico (21), se debe mencionar que estas patologías pueden ser provocadas directamente por las 
labores propias del cuidado, como también porque los cuidadores dejan de preocuparse de ellos mismos, situando en último lugar sus propias necesidades, lo que provoca disminución de la adherencia a los tratamientos médicos y estilos de vida poco saludables (22). Aunque también se debe considerar que la edad media de los cuidadores es mayor a 50 años, momento en que la población en general desarrolla estos problemas de salud.

El estudio expuso que la variable sobrecarga del cuidador no presenta correlación con las variables sociodemográficas, sólo resultó significativa la asociación con la variable instituciones que apoyan $(\mathrm{p}=0,042)$ y por lo tanto es factor predictivo de la sobrecarga, encontrándose que aquellos cuidadores que reciben ayuda de 2 o más instituciones tienen 8,6 puntos menos de sobrecarga que los que tienen una y ninguna institución que los apoyen, es decir existe una correlación con el apoyo social disponible. Esto es coincidente con estudios realizados por $\mathrm{Za}-$ rit et al. (33), quien analizó variables como la salud del cuidador, bienestar psicológico, ingresos, sexo, edad, nivel de educación, la vida social y la calidad de la relación entre el cuidador y la persona cuidada, la frecuencia de las visitas de otros familiares y el apoyo de los servicios sanitarios. Los resultados mostraron que sólo la frecuencia de visitas familiares se correlacionó significativamente con el nivel de sobrecarga $(r=0,48 ; p=<0,1)$, ninguna otra variable, inclusive la variable capacidad funcional del adulto mayor cuidado, presentó asociación.

Sin embargo, varios estudios revisados (2, $23,28,34)$ mostraban asociación de la sobrecarga con variables como sexo, tiempo y horas diarias dedicadas al cuidado, problemas de salud, nivel socioeconómico, escolaridad y creencia religiosa. Si bien es cierto, en este estudio no se puede comparar la variable capacidad funcional de la persona cuidada, ya que la población correspondió exclusivamente a cuidadores de personas adultos ma- yores con dependencia severa, permite plantear la búsqueda en futuras investigaciones de las causas que expliquen los altos niveles de sobrecarga encontrados.

El cuidado de las personas mayores no puede recaer sólo en un individuo, debe ser compartido con toda la familia, porque la carga y los costos que provoca son muy altos, y sólo se puede sobrellevar con la organización del trabajo familiar en forma igualitaria y equitativa.

El estudio fortalece el alcance de la enfermería comunitaria, como apoyo social y factor amortiguador de sobrecarga del cuidador familiar y ratifica la necesidad de mejorar el plan de apoyo al cuidador de personas con dependencia severa que existe en Chile, principalmente la cantidad de visitas domiciliarias que realiza el equipo multidisciplinario de salud comunitario, con intervenciones como educación, consejería, para proveer servicios centrados en las necesidades de los cuidadores, involucrando a familiares.

Confirma además la necesidad de contar con más instituciones comunitarias y gubernamentales que presten apoyo a estas personas con compromiso verdadero en la labor del cuidado y que se implementen estrategias a favor del cuidado familiar. El estudio aspira, además, a potenciar el surgimiento de iniciativas y proyectos innovadores de desarrollo social, que aumenten las redes de apoyo, como la creación de cargos comunitarios de enlace, desarrollo de una red telefónica o de internet para entregar una atención continuada y formulación de un programa de cuidados del cuidador, que incluya unidades de relevo y recreación para que las familias puedan afrontar de mejor forma el cuidado de un adulto mayor con dependencia severa. Los resultados permiten plantear líneas futuras de investigación, referente a la búsqueda de las causas que expliquen los altos niveles de sobrecarga encontrados, se podrían incluir variables importantes tales como: recursos de afrontamiento individual, resiliencia, significado que le otorgan al cuidado de 
un familiar, calidad de vida, los conocimientos que posea el cuidador, invisibilidad del trabajo de cuidador, autoestimación, niveles de ansiedad, depresión y funcionamiento familiar.

\section{REFERENCIAS}

1. Servicio Nacional del Adulto Mayor, Gobierno de Chile. Estudio situación de los Cuidadores Domiciliarios de los Adultos Mayores Dependientes con Pensión Asistencial. Chile. Informe final. [Internet]. Portal Mayores: España; 2007 [citado 20 octubre 2009]. Disponible en http:// www.imsersomayores.csic.es.

2. Pérez A. Dependencia, cuidado informal y función familiar. Análisis a través del modelo sociocultural de estrés y afrontamiento [Tesis Doctoral]. [Salamanca]: Universidad de Salamanca, 2008. [Internet] Portal Mayores. [citado 20 octubre 2009]. Disponible en http://www.imsersomayores.csic.es.

3. Waldow VR, Borges RF. The caregiving process in the vulnerability perspective. Rev Lat Am Enfermagem. 2008; 16(4): 765-771.

4. Díaz J, Rojas M. Cuidando al cuidador: efectos de un programa educativo. Aquichán. 2005; 9(1): 73-92.

5. Pinto N, Barrera L, Sánchez B. Reflexiones sobre el cuidado a partir del programa "Cuidando a los cuidadores". Aquichán. 2005; 5(1): 128-137. ISSN 1657-5997

6. Izal M, Montorio I, Díaz-Veiga P. Cuando las personas mayores necesitan ayuda: Guía para cuidadores y familiares. Madrid: INSERSO; 1997.

7. Flores JA, Adeva J, García MC, Gómez MP. Psicopatología de los cuidadores habituales de ancianos. Index Enferm. 1997; 3(1218): 261-272.

8. Sánchez B. Cómo cuidar un enfermo en casa. Aquichán 2005; 5(1): 162.
9. Casado D, López i Casasnovas G. Vejez, dependencia y cuidados de larga duración. Colección de estudios sociales. Barcelona: Ed. Fundación "la Caixa”. 200. p. 68-75.

10. Zavala M, Vidal D, Castro M, Quiroga P, Klassen G. Funcionamiento Social del Adulto Mayor. Cienc. enferm. 2006; XII(2): 53-62.

11. Mary L. Caregiver, Take Care. Geriatr Nurs. 2005; 26(3): 152-153.

12. Zarit SH, Davey A, Edwards AB, Femia EE, Jarrott SE. Family Caregiving: Research Findings and Clinical Implications. En: Bellack AS, Hersen M, editors y Edelstein BA, editor volumen. Comprehensive clinical psychology. Vol. 7, Clinical geropsychology. Oxford, UK: Elsevier Science; 1998. p. 499-523.

13. Consejo Europa. Recomendación $\mathrm{N}^{\circ} \mathrm{R}$ (98) 9 del Comité de Ministros a los Estados Miembros relativa a la dependencia; 1998 [citado 15 noviembre 2009]. Disponible en: http://www.imsersomayores. csic.es

14. Servicio Nacional del adulto Mayor. Estudio Nacional de la Dependencia en las Personas Mayores. [Internet] Santiago, Chile: INTA; 2010. p. 10-15. [citado 15 noviembre 2009]. Disponible en http:// www.senama.cl/

15. Araya A, Piwonka MA. Cuidados de enfermería en el adulto mayor. En: Marín Larraín PP, editor. Manual de geriatría y gerontología 2000 [Internet]. Chile: Pontificia Universidad Católica de Chile; 2000 [citado 20 noviembre 2009]. Disponible en: http://escuela.med.puc.cl/ publ/manualgeriatria/PDF/CuidadosEnfermeria.pdf

16. MINSAL. Plan de apoyo al cuidador de personas en situación de dependencia severa. Programa del Adulto Mayor. Unidad de Discapacidad y Rehabilitación: Chile; 1999. p. 7-11.

17. Zarit SH, Bottigi K, Gaugler JE. Stress and caregivers. En: Fink Get et al. edito- 
res. Encyclopedia of Stress. 2nd Edition. San Diego, CA: Academic Press; 2007. p. 416- 418.

18. Mockus S, Novielli K. A practical Guide to Caring for Caregivers. Am Fam Physician. 2009; 62: 2613-2621.

19. Tirado Pedregosa, G. Apoyo social en el cansancio del rol del cuidador. Evidencia. 2009; 6(25).

20. Lara L, Díaz M, Herrera E, Silveira P. Síndrome del "Cuidador" en una población atendida por equipo multidisciplinario de atención geriátrica. Rev Cubana Enferm. 2001; 17(2): 107-111.

21. Shultz R. Caregiver Burden. International Encyclopedia of the social \& Behavioral Sciences. 2004: 1476-1479.

22. Giraldo C, Franco G. Calidad de vida de los cuidadores familiares. Aquichan. 2006; 6(1): 38-53.

23. Ocampo J, Herrera J, Torres P, Rodríguez J, Loboa L, García C. Sobrecarga asociada con el cuidado de ancianos dependientes. Colomb. Med. 2007; 38: 40-46.

24. Pagano M, Gauvreau K. Fundamentos de Bioestadística. 2da Ed. México: Ed. Thomson-Learning; 2001.

25. Breinbauer $H$, Vásquez $H$, Mayanz $S$, Guerra C, Millán T. Validación en Chile de la Escala de Sobrecarga del Cuidador de Zarit en sus versiones original y abreviada. Rev Med Chil. 2009; 137(5): 657665.

26. Beauchamp T, Childress J. Principios de ética médica. 4ta Edición. Barcelona: Masson S.A.; 2002.

27. Guba E, Lincoln Y. Effective evaluation: improving the usefulness of evaluation results through responsive and naturalistic approaches. San Francisco: JosseyBass; 1981.

28. Chiou C, Chang H, Chen I, Wang H. Social support and caregiving circumstances as predictors of caregiver burden in Taiwan. Arch Gerontol Geriatr. 2009; 48: 419-424. Elsevier Inc.

29. Instituto Nacional de Estadísticas de Chile (INE). Características sociales y culturales. [citado 15 enero 2011].Disponible en: http://www.ine.cl/canales/sala_prensa/noticias/2004/may/not200504.php.

30. Ministerio de Desarrollo y Planificación. Encuesta CASEN, educación 2009. [citado 18 noviembre 2010]. Disponible en http://www.mideplan.cl/casen2009/educacion.php.

31. Dueñas E, Martínez A, Morales B, Muñoz C, Viáfara A, Herrera J. Síndrome del cuidador de adultos mayores discapacitados y sus implicancias psicosociales. Colomb. Med. 2006; 37(2), (S1): 31-38.

32. Deeken J, Taylor K, Mangan P, Yabroff R, Ingham J. Care for the Caregivers: A Review of Self-Report Instruments Developed to Measure the Burden, Needs, and Quality of Life of Informal Caregivers. J Pain Symptom Manage. 2003; 26(4): 922-953.

33. Zarit SH, Reever KE, Bach-Peterson J. Relatives of the impaired elderly: correlates of feelings of burden. Gerontologist. 1980; 20(6): 649-655.

34. Turner K. Social Support. Geriatr Nurs. 1988; 30(2): 336-338. 